\title{
Visible light-driven water oxidation with a ruthenium sensitizer and a cobalt-based catalyst connected with a polymeric platform $\uparrow$
}

\author{
Zeynep Kap ${ }^{a}$ and Ferdi Karadas (DD *ab
}

Received 5th November 2018, Accepted 11th December 2018

DOI: 10.1039/c8fd00166a

A facile synthesis for a photosensitizer-water oxidation catalyst (PS-WOC) dyad, which is connected through a polymeric platform, has been reported. The dyad assembly consists of a ruthenium-based chromophore and a cobalt-iron pentacyanoferrate coordination network as the water oxidation catalyst while poly(4-vinylpyridine) serves as the bridging group between two collaborating units. Photocatalytic experiments in the presence of an electron scavenger reveal that the dyad assembly maintains its activity for $6 \mathrm{~h}$ while the activity of a cobalt hexacyanoferrate and $\mathrm{Ru}(\mathrm{bpy})_{3}{ }^{2+}$ couple decreases gradually and eventually decays after a $3 \mathrm{~h}$ catalytic experiment. Infrared and XPS studies performed on the post-catalytic powder sample confirm the stability of the dyad during the catalytic process.

\section{Introduction}

Photocatalytic water splitting has been an attractive and promising research topic over the last two decades due to its potential contribution to sustainable and renewable energy development. ${ }^{1}$ The main objective with water splitting is to convert solar light into chemical energy and concurrently to produce hydrogen and oxygen. Since the demanding four-electron process of water oxidation is considered as the bottleneck of water splitting, research efforts have been centered on developing efficient assemblies for light-driven water oxidation catalysis.

In general, a photosensitizer (PS), which absorbs sunlight to create holes and electrons, collaborates with a water oxidation catalyst (WOC) to drive the water oxidation reaction in the presence of an electron scavenger. Recently dyads, in which the molecular PS and WOC are covalently coordinated to each other with

\footnotetext{
${ }^{a}$ Department of Chemistry, Bilkent University,06800 Ankara, Turkey.E-mail: karadas@fen.bilkent.edu.tr ${ }^{b}$ UNAM-Institute of Materials Science and Nanotechnology, Bilkent University, 06800 Ankara, Turkey $\dagger$ Electronic supplementary information (ESI) available: UV-Vis, FTIR, XPS, XRD, SEM, EDX characterizations, and details of photocatalytic studies. See DOI: 10.1039/c8fd00166a
} 
a suitable linker, have been constructed to develop dye-sensitized photoelectrochemical cells (DSPECs) to enhance the electron transfer and charge transport between molecular units and the semiconductor. ${ }^{2-5}$ Several $\mathrm{Ru}(\mathrm{II})$ based PS-WOC dyad assemblies have recently been coated on $\mathrm{TiO}_{2}$ to build dyesensitized DSPECs with promising faradaic efficiencies for $\mathrm{O}_{2}$ evolution. ${ }^{6,7}$ The catalytic efficiency of dyad systems was investigated in a homogeneous system as well. In a study by Thummel et al., a Ru-Ru dyad assembly showed a TON of 134 under $6 \mathrm{~h}$ illumination, which is much higher than its analogous intermolecular system with a TON of $6 .^{3}$ A follow-up study by Thummel et al. showed a TON of 68 under $1 \mathrm{~h}$ light illumination in the presence of sodium persulfate at pH 5.3. ${ }^{8}$ Sun et al. also prepared different PS-WOC assemblies, incorporating a ruthenium diimine chromophore and a ruthenium-based catalyst. ${ }^{9}$ The TON of the assembly was found to be 38 while the separate system showed a TON of 8 . In the majority of the dyad systems, ruthenium-based units have been preferred as both a WOC and a PS due to their strong light absorption, long excited state lifetimes, and high efficiencies. ${ }^{10}$ Implementing earth-abundant components, particularly for the catalytic site, still remains a significant challenge due to synthetic limitations.

The selection of a proper bridging group is one of the critical parameters for the design of an efficient dyad. Polymeric platforms have also been used for this purpose. ${ }^{10-14}$ Several studies indicate that enhanced catalytic efficiency observed on polymeric dyad systems is due to a hopping mechanism along the chain, which results in an intra-assembly electron/hole transfer., ${ }^{4,13}$ Waters et al. reported that an electrode-bound helical peptide PS-WOC assembly has a 10-fold improvement in its catalytic activity compared to its analogous homogeneous system. ${ }^{12}$ It has been shown that intra-assembly electron transfer is a key parameter to enhance efficiency and for aligning the distance between units for optimum electron transfer rates. ${ }^{12,13}$ Hisaeda et al. emphasized that an assembly with a polymer linkage can also efficiently work even under diluted conditions by fixation of each functional group in the same polymeric unit, thus providing a close distance for electron transfer. ${ }^{15}$ In the presence of a polymeric support, stability of the system is also expected to increase by preventing photodecomposition of the photosensitizer. ${ }^{15,16}$

In this study, we present a novel heterogeneous PS-WOC dyad by using poly(4vinylpyridine) (P4VP) as a bridging platform between a ruthenium chromophore and cobalt-based Prussian blue analogue (PBA). Cobalt hexacyanometalates have recently been demonstrated as promising water oxidation catalysts due to their high catalytic activities, robustness, and stabilities at a wide range of $\mathrm{pH}$ ( 1 to 13). ${ }^{17-24}$ Therefore, the use of a CoFe-PBA as a WOC rather than a Ru-based one will be a step forward in the development of entirely earth abundant dyads. P4VP has recently been used to prepare a Co-Fe coordination polymer for water oxidation electrocatalysis by our group..$^{25}$ The study involved the coordination of $\mathrm{Fe}(\mathrm{CN})_{5}$ groups to the pyridyl groups of P4VP yielding a robust precursor for the synthesis of amorphous PBAs. In another study, a Co-P4VP assembly has successfully been prepared and found to be an efficient metallopolymer for water reduction electrocatalysis. ${ }^{26}$ Given the successful utilization of P4VP for catalytic applications, herein, we propose a synthetically facile dyad, wherein the ruthenium-based molecular photosensitizer is connected to a Prussian blue type water oxidation catalyst through a P4VP platform. Photocatalytic water oxidation 
performance has been investigated in comparison with a cobalt-based PBA. Characterization techniques have also been performed to evaluate its stability.

\section{Experimental}

\section{Starting materials}

cis-Bis(2,2'-bipyridine)dichlororuthenium(II) hydrate (Acros Organics, 97\%), poly(4-vinylpyridine) (Sigma-Aldrich, $\mathrm{MW} \sim 60000$ ), $\mathrm{AgNO}_{3}$ (Sigma-Aldrich, $\geq 99.0$ ), $\mathrm{Na}_{2}\left[\mathrm{Fe}^{\mathrm{III}}(\mathrm{CN})_{5} \mathrm{NO}\right] \cdot 2 \mathrm{H}_{2} \mathrm{O}$ (Alfa Aesar, 98\%), and $\mathrm{NaOH}$ (Sigma-Aldrich, 98-100.5\%) were used. All the solvents were analytical grade and reagents received were used without any further processing. Millipore deionized water (resistivity: $18 \mathrm{~m} \Omega \mathrm{cm}$ ) was used for all experiments that required water.

\section{Synthetic procedures}

General procedure for synthesis of [Ru-P4VP]. At room temperature, $700.0 \mathrm{mg}$ (1.445 mmol) cis-[Ru(bpy $\left.)_{2} \mathrm{Cl}_{2}\right]$ and $490.9 \mathrm{mg}(2.890 \mathrm{mmol}) \mathrm{AgNO}_{3}$ are mixed in $100 \mathrm{~mL}$ methanol according to the modified literature. ${ }^{27}$ After $1 \mathrm{~h}$ vigorous stirring, the precipitated layer of $\mathrm{AgCl}$ was filtered through a Celite ${ }^{\circledR}$ filter and removed. $\left[\mathrm{Ru}(\mathrm{bpy})_{2}\left(\mathrm{H}_{2} \mathrm{O}\right)_{2}\right]\left(\mathrm{NO}_{3}\right)_{2}$ filtrate was evaporated by a rotary evaporator. $\left[\mathrm{Ru}(\mathrm{bpy})_{2}\left(\mathrm{H}_{2} \mathrm{O}\right)_{2}\right]\left(\mathrm{NO}_{3}\right)_{2}$ was added to the solution of 6-fold molar excess of poly $(4-$ vinylpyridine) which was dissolved in $200 \mathrm{~mL} 4: 1$ ethanol/water. The mixture was refluxed in the dark for $48 \mathrm{~h}$ under constant stirring. Completion of the product was monitored by UV-Vis spectroscopy. The resulting solution was evaporated by a rotary evaporator, dissolved in ethanol, and precipitated by ethyl ether. ${ }^{28}$ The precipitate was filtered and rinsed with cold water and ethyl ether. Throughout the article, the abbreviation [Ru-P4VP] will be used for the $\left[\mathrm{Ru}(\mathrm{bpy})_{2}(\mathrm{P} 4 \mathrm{VP})_{6}\right]$.

General procedure for synthesis of the Fe precursor. $\mathrm{Na}_{3}\left[\mathrm{Fe}^{\mathrm{II}}(\mathrm{CN})_{5} \mathrm{NH}_{3}\right] \cdot 3 \mathrm{H}_{2} \mathrm{O}$ was used as the Fe precursor. According to the procedure in literature with slight modifications, ${ }^{25,29} 30 \mathrm{~g}$ of $\mathrm{Na}_{2}\left[\mathrm{Fe}^{\mathrm{III}}(\mathrm{CN})_{5} \mathrm{NO}\right] \cdot 2 \mathrm{H}_{2} \mathrm{O}$ and $4 \mathrm{~g} \mathrm{NaOH}$ were mixed in $120 \mathrm{~mL}$ of water under constant stirring. Throughout the experiment, the temperature was kept below $10{ }^{\circ} \mathrm{C}$. After obtaining a homogenous solution, $25 \%$ (v/v) $\mathrm{NH}_{4} \mathrm{OH}$ solution was added until saturation, followed by the addition of cold methanol until a yellow color was obtained. The product was recrystallized using $\mathrm{NH}_{4} \mathrm{OH}$ and $\mathrm{CH}_{3} \mathrm{OH}$ solutions. After vacuum filtration, the resulting precipitate was dried in a vacuum oven overnight at $25{ }^{\circ} \mathrm{C}$. IR $\left(\mathrm{cm}^{-1}\right)$ : 3300(b), 2135(s), 2009(m), 1642(m), 1621(m), 1257(m), 569(m).

General procedure for synthesis of [Ru-P4VP-Fe]. The [Ru-P4VP] was dissolved in methanol and the precursor was added according to a $1: 2 \mathrm{Ru} / \mathrm{Fe}$ ratio. The solution was kept in the dark under constant stirring for 5 days. Cold $[\mathrm{Ru}-$ P4VP-Fe] solution was centrifuged with water three times and the solution was discarded. The complex was dried after washing with acetone in a vacuum desiccator. Throughout the manuscript, the abbreviation [Ru-P4VP-Fe] will be used for the $\left[\mathrm{Ru}(\mathrm{bpy})_{2}(\mathrm{P} 4 \mathrm{VP})_{6}\right]-\mathrm{Fe}(\mathrm{CN})_{5}$ assembly.

General procedure for synthesis of [Ru-P4VP-CoFe]. Cobalt(II) acetate tetrahydrate was used as the Co precursor. [Ru-P4VP-Fe] and the Co precursor were mixed in a $1: 1$ acetonitrile/water solution. The Co precursor was added according to the $3: 2 \mathrm{Co} / \mathrm{Fe}$ stoichiometric ratio. The solution was kept in the dark under constant stirring for 2 days following evaporation by a rotary evaporator. 


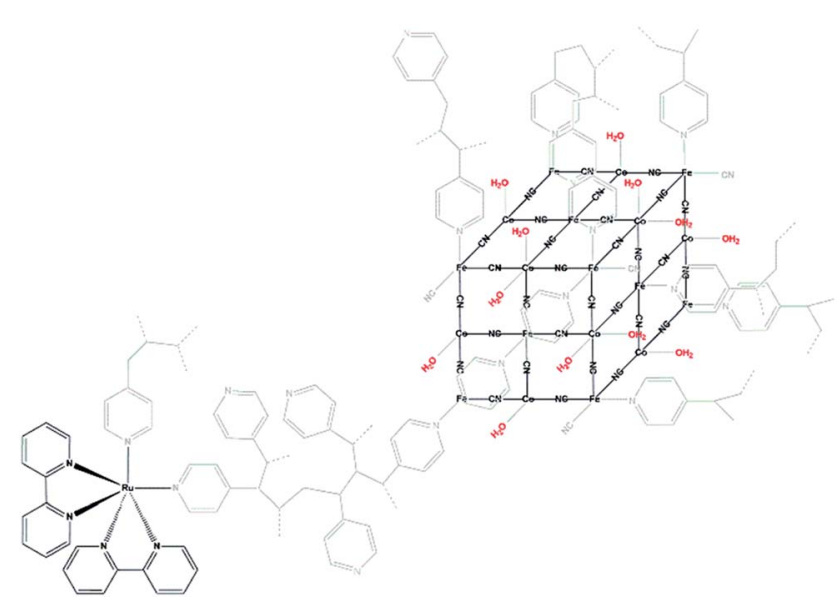

Fig. 1 Proposed structure of the ruthenium chromophore and cobalt-based PBA dyad, incorporating poly(4-vinylpyridine).

Throughout the article, the abbreviation [Ru-P4VP-CoFe] will be used for the $\left[\mathrm{Ru}(\mathrm{bpy})_{2}(\mathrm{P} 4 \mathrm{VP})_{6}\right]-\mathrm{CoFe}(\mathrm{CN})_{5}$ assembly. The proposed structure of the assembly is shown in Fig. 1.

\section{Photochemical setup}

The oxygen amount was measured with GC (Agilent 7820A, Molesieve GC column $(30 \mathrm{~m} \times 0.53 \mathrm{~mm} \times 25 \mu \mathrm{m}))$ thermostatted at $40{ }^{\circ} \mathrm{C}$ which was equipped with a TCD detector thermostatted at $100{ }^{\circ} \mathrm{C}$ (Ar as carrier gas). Oxygen evolution was calibrated with a pressure transducer (Omega PXM409-002BAUSBH). The solar light simulator (Sciencetech, SLB-300B, $300 \mathrm{~W}$ Xe lamp, AM 1.5 global filter) was calibrated to 1 sun $\left(100 \mathrm{~mW} \mathrm{~cm}^{-2}\right)$. Experimental setup is shown in ESI $\dagger$ and explained in detail.

\section{Results and discussion}

\section{Characterization}

$\mathrm{Ru}(\mathrm{bpy})_{2} \mathrm{Cl}_{2}$ exhibits two characteristic bands at 526 and $283 \mathrm{~nm}$, which are assigned to metal-to-ligand charge transfer (MLCT) and ligand centered $\pi-\pi^{*}$ (LC) transitions, respectively. On the other hand, a blue shift to 465 (with a shoulder at $435 \mathrm{~nm}$ ) is obtained for [Ru-P4VP] verifying the complex formation, which are in good accordance with absorption profiles of trisbipyridyl-ruthenium(II) complexes (Fig. 2)..$^{30-32}$ These bands were also observed for [Ru-P4VP$\mathrm{Fe}]$ and [Ru-P4VP-CoFe], which indicates that the ruthenium ion is surrounded with pyridyl groups in all compounds and that the ruthenium site in [Ru-PVPCoFe] could serve as a chromophore to utilize visible light (Fig. S1†).

The infrared spectrum of [Ru-P4VP] exhibits two major bands at $1417 \mathrm{~cm}^{-1}$ and $1597 \mathrm{~cm}^{-1}$, which are attributed to the $\mathrm{C}=\mathrm{C}_{\text {ring }}$ and $\mathrm{C}=\mathrm{N}_{\text {ring }}$ of pyridyl rings (Fig. S2 $\dagger$ ). ${ }^{25,33}$ An additional band in the $2000-2200 \mathrm{~cm}^{-1}$ range is observed for $[\mathrm{Ru}-\mathrm{P} 4 \mathrm{VP}-\mathrm{Fe}]$, which corresponds to the $\mathrm{C} \equiv \mathrm{N}$ stretches of the $\left[\mathrm{Fe}(\mathrm{CN})_{5}\right]$ fragment (Fig. 3). The relatively small peak at $2103 \mathrm{~cm}^{-1}$ is a result of the partial oxidation 


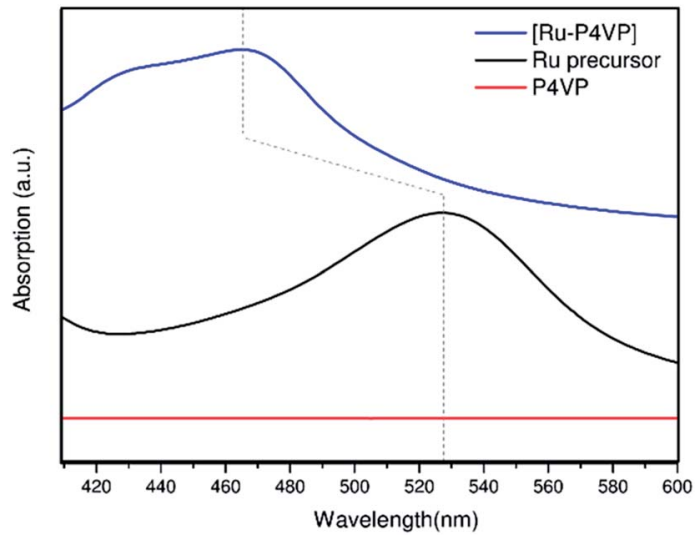

Fig. 2 UV-Vis spectra of P4VP (red), Ru precursor (black), and [Ru-P4VP] (blue) in ethanol.

of the iron sites to $\mathrm{Fe}^{3+}$. The reaction with $\mathrm{Co}^{2+}$ leads to a shift to higher frequency due to the formation of the $\mathrm{Fe}-\mathrm{CN}-\mathrm{Co}$ coordination mode. ${ }^{25,34}$

XPS studies indicate an observable change in the binding energy of the $\mathrm{Ru} 3 \mathrm{~d}_{5 /}$ ${ }_{2}$ signal for [Ru-P4VP] compared with that of $\mathrm{Ru}(\mathrm{bpy})_{2} \mathrm{Cl}_{2}$ as a result of the replacement of chloride groups with pyridyl ones. $\mathrm{Ru} 3 \mathrm{~d}_{5 / 2}$ signals are considered for comparison due to overlap of the $\mathrm{Ru} 3 \mathrm{~d}_{3 / 2}$ and $\mathrm{C} 1 \mathrm{~s}$ signals (Fig. 4). Besides, the $\mathrm{Ru} 3 \mathrm{~d}$ signals in [Ru-P4VP], [Ru-P4VP-Fe], and [Ru-P4VP-CoFe] samples are similar suggesting no significant changes in the coordination sphere and oxidation state of the ruthenium site. The slight change in the Fe $2 \mathrm{p}$ band in [Ru-P4VP$\mathrm{Fe}]$ is attributed to the partial oxidation of $\mathrm{Fe}^{3+/ 2+}$. Two shoulder bands observed at $\sim 711.51 \mathrm{eV}$ and $\sim 725.21 \mathrm{eV}$ in the Fe 2p signals of [Ru-P4VP-Fe] can also be attributed to the aforementioned partial oxidation process (Fig. S3†). Such oxidation is commonly observed in pentacyanoferrate chemistry, and the results are in good agreement with FTIR spectra, which reveals a shoulder band in the cyanide region at $2103 \mathrm{~cm}^{-1}$ for [Ru-P4VP-Fe]. Fe 2p signals for [Ru-P4VP-Fe] are

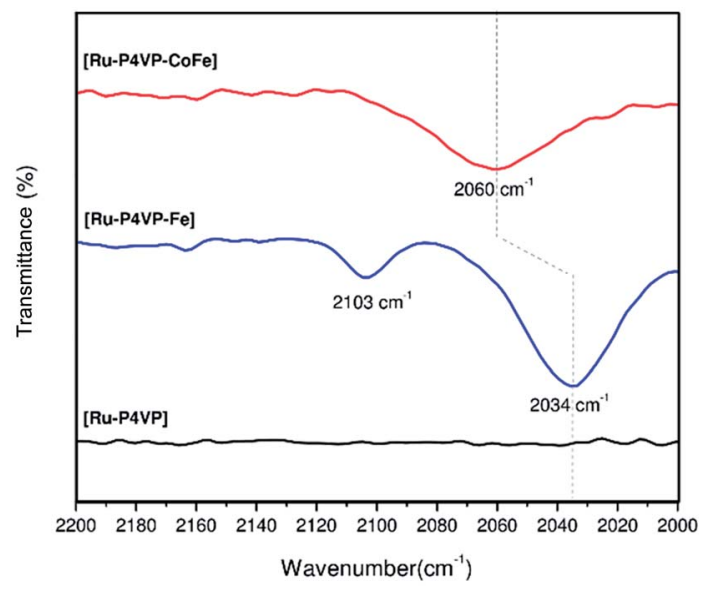

Fig. 3 FTIR spectra of [Ru-P4VP], [Ru-P4VP-Fe], and [Ru-P4VP-CoFe]. 


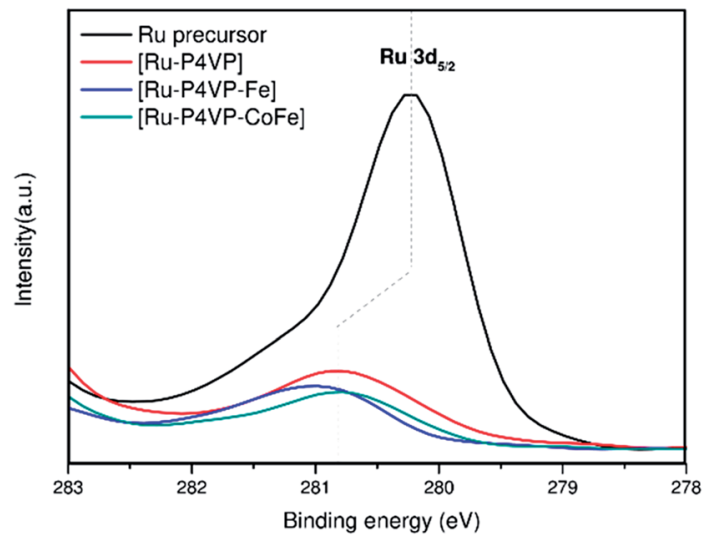

Fig. 4 XPS spectra of the Ru $3 d_{5 / 2}$ signals of [Ru-P4VP-CoFe] (green), [Ru-P4VP-Fe] (blue), [Ru-P4VP] (red), and the Ru precursor (black).

observed at around $708.69 \mathrm{eV}$ and $722.69 \mathrm{eV}$, which are assigned to $\mathrm{Fe} 2 \mathrm{p}_{3 / 2}$ and $\mathrm{Fe}$ $2 \mathrm{p}_{1 / 2}$, respectively. The signals of [Ru-P4VP-Fe] and [Ru-P4VP-CoFe] correspond well with those of the Fe precursor. Broad features observed in [Ru-P4VP-CoFe] indicate the presence of multiple oxidation states of iron sites. The $\mathrm{N} 1 \mathrm{~s}$ band of [Ru-P4VP] corresponds to the pyridyl groups of P4VP and the bipyridyl groups of the ruthenium fragment (Fig. S4 $\dagger$ ). A slight shift in the binding energy of $[\mathrm{Ru}-$ $\mathrm{P} 4 \mathrm{VP}]$ compared with the Ru precursor is attributed to an increase in the electron density of the pyridyl ring because of the $\pi$ back-bonding interaction between ruthenium and the pyridyl groups of P4VP. A similar response is also observed for [Ru-P4VP-Fe] and [Ru-P4VP-CoFe]. A band observed at higher binding energies reveals the presence of nitrate anions for [Ru-P4VP], which are available to provide charge balance. ${ }^{35}$ The cobalt precursor exhibits Co $2 \mathrm{p}_{3 / 2}$ and $2 \mathrm{p}_{1 / 2}$ peaks at $781.09 \mathrm{eV}$ and $796.96 \mathrm{eV}$, respectively. Similarly, those of [Ru-P4VP-CoFe] are positioned at $781.01 \mathrm{eV}$ and $796.54 \mathrm{eV}$, suggesting the presence of cobalt ions with $\mathrm{a}+2$ oxidation state (Fig. 5). Furthermore, the cobalt region of [Ru-P4VP-CoFe]

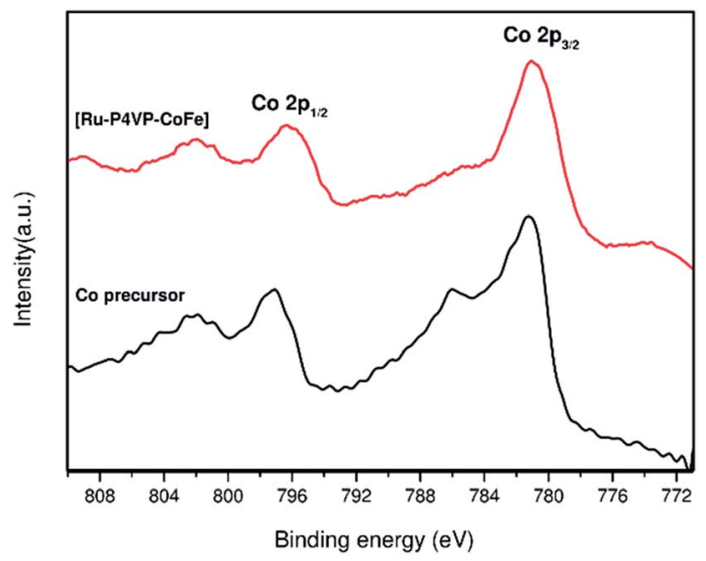

Fig. 5 XPS spectra of the Co 2p signals of the Co precursor, and [Ru-P4VP-CoFe]. 
contains satellite signals at binding energies approximately $5 \mathrm{eV}$ higher than the principal signals.

$\mathrm{XRD}$ analysis conducted on [Ru-P4VP-CoFe] powder reveals characteristic peaks of PB structure. The broad nature of the peaks implies the formation of small PB structures due to the polymeric moiety (Fig. S5†). The structural morphology was also confirmed by SEM studies (Fig. S6†). Cubic structures with particle sizes of around $50 \mathrm{~nm}$ were observed. EDX studies also confirm the presence of $\mathrm{Ru}, \mathrm{Co}, \mathrm{Fe}$, and a small quantity of Na yielding a rough molecular formula of $\mathrm{Na}_{0.96} \mathrm{Co}_{2.86}\left[\mathrm{Fe}(\mathrm{CN})_{5}\right]_{2.13}-[\mathrm{P} 4 \mathrm{VP}]_{6}-\left[\mathrm{Ru}(\mathrm{bpy})_{2}\right] \mathrm{Cl}_{2.29}$ (Fig. S7 $\dagger$ ). It should be noted that an average of two out of six pyridyl groups are estimated to react with $\left[\mathrm{Ru}(\mathrm{bpy})_{2}\right]$ fragments $^{21,22}$ while the ratio is $1: 3$ for $\left[\mathrm{Fe}(\mathrm{CN})_{5}\right] /$ pyridyl. SEM studies performed on different regions of the powder sample indicate the lack of a uniform stoichiometric ratio between metal ions. Such a non-uniform distribution can be explained by both the nonstoichiometric nature of PBAs ${ }^{36}$ and their integration with a non-uniform Ru-polymer system. Furthermore, the amount of chloride ions is higher in regions where ruthenium is more abundant. A similar trend was also observed for sodium atoms with respect to cobalt and iron atoms, which suggests that $\left[\mathrm{Ru}(\mathrm{bpy})_{2}\right]$ and CoFe PBA exhibit a cationic and anionic nature, respectively. Thus, chloride ions are present to provide the charge balance in regions where ruthenium ions are in excess, while $\mathrm{Na}$ ions serve a similar purpose for PB structures. Overall, the characterization studies conclude that $\mathrm{P} 4 \mathrm{VP}$ is coordinated to both $\left[\mathrm{Ru}(\mathrm{bpy})_{2}\right]$ fragments and cubic PB structures.

\section{Catalytic performance}

Photocatalytic studies were performed on a suspension solution containing [RuP4VP-CoFe] powder and $\mathrm{Na}_{2} \mathrm{~S}_{2} \mathrm{O}_{8}$ as the electron scavenger, at $\mathrm{pH}$ 7. Photocatalytic experiments were also performed with a previously studied cobalt hexacyanoferrate (labeled as Co-Fe PBA throughout the manuscript) in the presence of a $\left[\mathrm{Ru}(\mathrm{bpy})_{3}\right]^{2+} / \mathrm{S}_{2} \mathrm{O}_{8}{ }^{2-}$ couple for comparison under similar conditions. ${ }^{17}$ In both experiments, the quantity of $\mathrm{O}_{2}$ in the gas-tight set-up was measured before and after with gas chromatography. Blank measurements without a catalyst, a chromophore, and an electron scavenger were also carried out under the same conditions.

The experiment for [Ru-P4VP-CoFe] was performed for six cycles with the same batch while the experiment for the $\left[\mathrm{Ru}(\mathrm{bpy})_{3}\right]^{2+}$ and Co-Fe PBA couple was performed for three cycles. The $\left[\mathrm{Ru}(\mathrm{bpy})_{3}\right]^{2+}$ and Co-Fe PBA curve yields a turnover frequency of $4.5 \times 10^{-4} \mathrm{~s}^{-1}$, which is in good agreement with the previous study. ${ }^{17}$ The catalytic activity of $\left[\mathrm{Ru}(\mathrm{bpy})_{3}\right]^{2+}$ and Co-Fe PBA system decreases gradually. In the final cycle, the number of moles of $\mathrm{O}_{2}$ produced reached the value of the blank measurement $\left(\left[\mathrm{Ru}(\mathrm{bpy})_{3}\right]^{2+}\right.$ and Co-Fe PBA without an electron scavenger, Fig. S8 $\dagger$ ), which is attributed to the decomposition of $\left[\mathrm{Ru}(\mathrm{bpy})_{3}\right]^{2+}$ complex under photocatalytic conditions. ${ }^{17}$

The photocatalytic water oxidation performance of PBAs was previously investigated by Galán-Mascarós et al. with characterization studies performed on the post-catalytic sample. ${ }^{17}$ The origin of the decaying trend was found to be due to the photodecomposition of the $\mathrm{Ru}$ chromophore by releasing its pyridyl groups. These groups then poison the catalyst by coordinating to catalytic cobalt sites. On the other hand, [Ru-P4VP-CoFe] maintained its catalytic activity for six 
cycles. TOF ranges from $3 \times 10^{-4} \mathrm{~s}^{-1}$ to $6 \times 10^{-4} \mathrm{~s}^{-1}$ throughout these cycles. The slight variation in the catalytic performance can be attributed to the change in the morphology of the powder sample during the catalytic process and/or to the rough approximations made for the determination of TOF. For example, all cobalt sites are assumed to be catalytically active in the estimation of TON and TOF for [Ru-P4VP-CoFe]. Given a particle size of $50 \mathrm{~nm}$ for cubic-shaped particles obtained by SEM image (Fig. S6†), a rough calculation indicates that only around $3 \%$ of the cobalt sites are on the surface and active. Thus, the changes in TOF during each cycle are well within the error range (Fig. 6).

A TON of 11 is obtained after six cycles under a total of $6 \mathrm{~h}$ light illumination while the $\mathrm{Ru}(\mathrm{bpy})_{3}{ }^{2+}$ and Co-Fe PBA system achieved only a TON of 2 after three cycles in a $3 \mathrm{~h}$ period. The results show that the ruthenium complex in [Ru-P4VPCoFe] serves as a chromophore similar to $\mathrm{Ru}(\mathrm{bpy})_{3}{ }^{2+}$ and coupling it with a heterogeneous catalyst enhances its stability dramatically (Fig. 7).

\section{Post-catalytic characterization}

The stability of the catalyst has been investigated in detail by performing XPS and infrared studies on the post-catalytic powder sample. The suspension solution was filtered, washed several times with distilled water, and dried in a vacuum desiccator to obtain the post-catalytic powder sample.

The XPS analysis of Co 2p and O 1s binding energies in the pristine and postcatalytic samples were carried out for [Ru-P4VP-CoFe]. The spectra of the Co $2 \mathrm{p}$ bands exhibit similar Co $2 \mathrm{p}_{3 / 2}$, Co $2 \mathrm{p}_{1 / 2}$, and satellite bands (Fig. S9 $\dagger$ ). Moreover, a lack of peaks below $780 \mathrm{eV}$ rules out the decomposition of Co-Fe PBA to a possible catalytically active oxide species. ${ }^{26}$ XPS of the $\mathrm{O} 1 \mathrm{~s}$ region was conducted to confirm that there is no decomposition of the metal-coordinated clusters which might lead to a mixed metal oxide. Analysis of the $\mathrm{O} 1 \mathrm{~s}$ region clearly shows that there is no cobalt oxide species, which typically have binding energies lower than $530 \mathrm{eV}$, and peaks observed around $531 \mathrm{eV}$ are only due to surface-adsorbed oxygen species (Fig. S10†). ${ }^{25}$ Based on the comparison of the Ru $3 \mathrm{~d}_{5 / 2}$ band of pristine and post catalytic [Ru-P4VP-CoFe], possible formation of $\mathrm{RuO}_{2}$ can be ruled out (Fig. S11†). ${ }^{37}$ It should also be noted that a slight

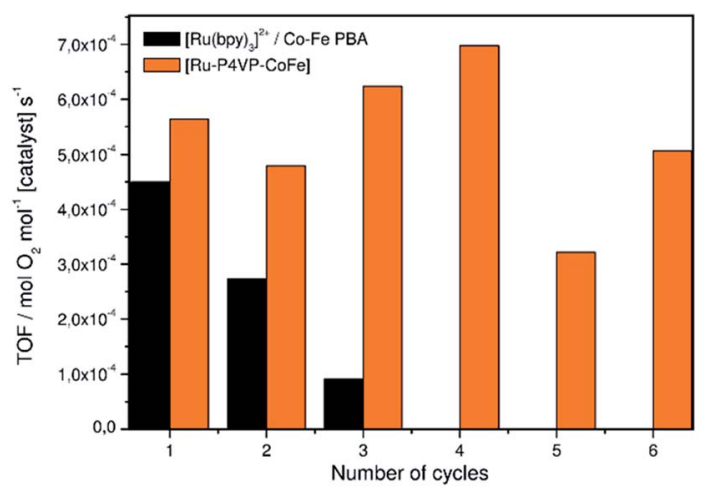

Fig. 6 TOF vs. number of cycles comparison of [Ru-P4VP-CoFe] (orange bar) and $\left[\mathrm{Ru}(\mathrm{bpy})_{3}\right]^{2+} / \mathrm{Co}-\mathrm{Fe} \mathrm{PBA}$ system (black bar). Each cycle duration is $1 \mathrm{~h}$. 


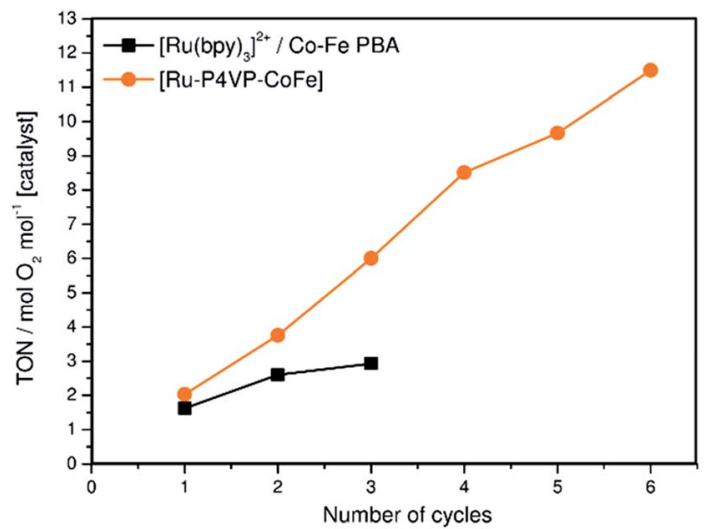

Fig. 7 TON vs. number of cycles comparison of [Ru-P4VP-CoFe] (orange, $\mathbf{0}$ ) and $\left[\mathrm{Ru}(\mathrm{bpy})_{3}\right]^{2+} / \mathrm{Co}-\mathrm{Fe}$ PBA system (black,

broadening in the $\mathrm{O} 1 \mathrm{~s}$ peak and a slight shift in the $\mathrm{Ru} 3 \mathrm{~d}_{3 / 2}$ peak has been observed for the post-catalytic sample, which could be attributed to the formation of a trace amount of ruthenium oxide species.

The cyanide stretch of the post-catalytic sample shifts to higher wavenumbers, which is attributed to partial oxidation of $\mathrm{Co}^{2+}$ to $\mathrm{Co}^{3+}$ during photoexcitation (Fig. S12 $\dagger$ ). As pointed out by Galán-Mascarós et al., this change could also be due to linkage isomerism (CN bond flipping). ${ }^{17}$ Furthermore, two major bands of the pristine sample at $1417 \mathrm{~cm}^{-1}$ and $1597 \mathrm{~cm}^{-1}$, which are attributed to $\mathrm{C}=\mathrm{C}_{\text {ring }}$ and $\mathrm{C}=\mathrm{N}_{\text {ring }}$ of pyridyl rings, were observed also for the post-catalytic sample (Fig. 8).

Although the [Ru-P4VP-CoFe] assembly showed enhanced activity and stability compared with its uncoordinated analogue system, comparison should also be made with dyad systems reported in the literature. Photocatalytic activity is modest in comparison with the Ru-based dyads reported by Thummel et al. (TON of 134 and 68), ${ }^{3,8}$ and the trinuclear ruthenium assembly studied by Sun et al. (TON of 38). ${ }^{9}$ [ [Ru-P4VP-CoFe] exhibits a higher TON than a molecular

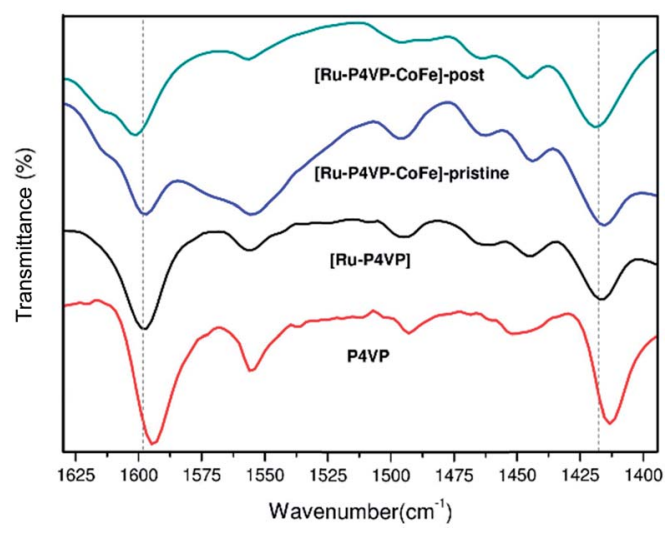

Fig. 8 FTIR spectra of P4VP, [Ru-P4VP], [Ru-P4VP-CoFe], and [Ru-P4VP-CoFe] after six catalytic cycle. 
cobalt-based dyad (TON of 5) reported by Sun et al. ${ }^{38}$ The stability of molecular dyads have generally been investigated with relatively short-term experiments, which are in the order of minutes. This study, however, represents a dyad that maintains its activity during a $6 \mathrm{~h}$ photocatalytic experiment. The stability of [Ru$\mathrm{P} 4 \mathrm{VP}-\mathrm{CoFe}$ ] can be attributed to the inherent robustness of the rigid cyanide network. It is also important to underline that no studies have been reported so far on the re-usability of dyads, and therefore, this study is novel in the line of techniques to analyze catalytic performance of dyads.

Polymeric dyad assemblies presented in the literature are investigated in DSPEC systems where the assembly is anchored to a semiconductor. In such studies, the activities of the dyads are analyzed in different experimental conditions and are reported in terms of current densities and faradaic efficiencies. For this reason, fair comparison of the [Ru-P4VP-CoFe] system with polymeric dyads reported cannot be made.

\section{Conclusions}

Overall, a novel heterogeneous PS-WOC dyad, incorporating poly(4vinylpyridine) as a bridge between a ruthenium chromophore and a cobaltbased PBA, was presented. The structure of each of the intermediate products ([Ru-P4VP] and [Ru-P4VP-Fe]) and that of the final product [Ru-P4VPCoFe], was monitored and elucidated with infrared, UV-Vis, and XPS studies. EDX studies revealed the formation of a non-stoichiometric compound, wherein the atomic ratio of $\mathrm{Ru}$ to Fe atoms varies from 1.87 to 2.56, the average of which yields a rough molecular formula of $\mathrm{Na}_{0.96} \mathrm{Co}_{2.86}\left[\mathrm{Fe}(\mathrm{CN})_{5}\right]_{2.13}-[\mathrm{P} 4 \mathrm{VP}]-$ $\left[\mathrm{Ru}(\mathrm{bpy})_{2}\right] \mathrm{Cl}_{2.29}$. SEM and XRD studies indicate the formation of small Prussian blue cubic structures with a size of around $50 \mathrm{~nm}$. Catalytic performance of the dyad was investigated under $1 \mathrm{~h}$ light illumination, and oxygen evolution was measured with GC where a pressure transducer was used as a supporting device to sense the pressure during the photocatalytic experiment. The dyad showed slightly higher catalytic activity (a TOF of $5.6 \times 10^{-4} \mathrm{~s}^{-1}$ ) compared with the relevant multi-component system (a TOF of $4.5 \times 10^{-4} \mathrm{~s}^{-1}$ ), which could be attributed to the increase in the number of active cobalt sites on the surface due to change in the morphology or improvement in the activity of catalytically active cobalt sites or a combination of both. Moreover, [Ru-P4VP-CoFe] maintained a steady activity for six cycles. Characterization studies performed on the pristine and post-catalytic powder samples confirmed the stability of the assembly under harsh photocatalytic conditions. This result indicates that rigid PB structures serve not only as a water oxidation catalyst but also as a protective layer for the chromophore. Therefore, immobilization of chromophores via coordination to cyanide-based frameworks could be a viable approach for the development of robust and active dyad assemblies for photocatalytic water oxidation. The diversity, easy synthesis, and remarkable stability of cyanide-based frameworks make them ideal candidates for the development of dye-sensitized photoanodes for water oxidation.

\section{Conflicts of interest}

There are no conflicts to declare. 


\section{Acknowledgements}

This work is supported by the Scientific and Technological Research Council of Turkey (TUBITAK), grant number 215Z249. Ferdi Karadas thanks TÜBA-GEBIP and BAGEP for young investigator awards.

\section{Notes and references}

1 J. L. Dempsey, A. J. Esswein, D. R. Manke, J. Rosenthal, J. D. Soper and D. G. Nocera, Inorg. Chem., 2005, 44, 6879-6892.

2 J. J. Concepcion, J. W. Jurss, P. G. Hoertz and T. J. Meyer, Angew. Chem., Int. Ed., 2009, 48, 9473-9476.

3 N. Kaveevivitchai, R. Chitta, R. Zong, M. El Ojaimi and R. P. Thummel, J. Am. Chem. Soc., 2012, 134, 10721-10724.

4 D. L. Ashford, M. K. Gish, A. K. Vannucci, M. K. Brennaman, J. L. Templeton, J. M. Papanikolas and T. J. Meyer, Chem. Rev., 2015, 115, 13006-13049.

5 M. Yamamoto, L. Wang, F. Li, T. Fukushima, K. Tanaka, L. Sun and H. Imahori, Chem. Sci., 2016, 7, 1430-1439.

6 B. D. Sherman, D. L. Ashford, A. M. Lapides, M. V. Sheridan, K. R. Wee and T. J. Meyer, J. Phys. Chem. Lett., 2015, 6, 3213-3217.

7 B. D. Sherman, Y. Xie, M. V. Sheridan, D. Wang, D. W. Shaffer, T. J. Meyer and J. J. Concepcion, ACS Energy Lett., 2017, 2, 124-128.

8 L. Kohler, N. Kaveevivitchai, R. Zong and R. P. Thummel, Inorg. Chem., 2014, 53, 912-921.

9 F. Li, Y. Jiang, B. Zhang, F. Huang, Y. Gao and L. Sun, Angew. Chem., Int. Ed., 2012, 51, 2417-2420.

10 G. Leem, B. D. Sherman and K. S. Schanze, Nano Convergence, 2017, 4, 37.

11 J. Jiang, B. D. Sherman, Y. Zhao, R. He, I. Ghiviriga, L. Alibabaei, T. J. Meyer, G. Leem and K. S. Schanze, ACS Appl. Mater. Interfaces, 2017, 9, 19529-19534.

12 D. M. Ryan, M. K. Coggins, J. J. Concepcion, D. L. Ashford, Z. Fang, L. Alibabaei, D. Ma, T. J. Meyer and M. L. Waters, Inorg. Chem., 2014, 53, 8120-8128.

13 S. E. Bettis, D. M. Ryan, M. K. Gish, L. Alibabaei, T. J. Meyer, M. L. Waters and J. M. Papanikolas, J. Phys. Chem. C, 2014, 118, 6029-6037.

14 Z. Fang, A. Ito, H. Luo, D. L. Ashford, J. J. Concepcion, L. Alibabaei and T. J. Meyer, Dalton Trans., 2015, 44, 8640-8648.

15 H. Shimakoshi, M. Nishi, A. Tanaka, K. Chikama and Y. Hisaeda, Chem. Commun., 2011, 47, 6548-6550.

16 G. Leem, Z. A. Morseth, K.-R. Wee, J. Jiang, M. K. Brennaman, J. M. Papanikolas and K. S. Schanze, Chem.-Asian J., 2016, 11, 1257-1267.

17 S. Goberna-Ferrón, W. Y. Hernández, B. Rodríguez-García and J. R. GalánMascarós, ACS Catal., 2014, 4, 1637-1641.

18 E. P. Alsaç, E. Ülker, S. V. K. Nune, Y. Dede and F. Karadas, Chem.-Eur.J., 2018, 24, 4856-4863.

19 L. Han, P. Tang, Á. Reyes-Carmona, B. Rodríguez-García, M. Torréns, J. R. Morante, J. Arbiol and J. R. Galan-Mascaros, J. Am. Chem. Soc., 2016, 138, 16037-16045.

20 Y. Yamada, K. Oyama, R. Gates and S. Fukuzumi, Angew. Chem., Int. Ed., 2015, 54, 5613-5617. 
21 Y. Isaka, K. Oyama, Y. Yamada, T. Suenobu and S. Fukuzumi, Catal. Sci. Technol., 2016, 6, 681-684.

22 Y. Yamada, K. Oyama, T. Suenobu and S. Fukuzumi, Chem. Commun., 2017, 53, 3418-3421.

23 F. S. Hegner, D. Cardenas-Morcoso, S. Giménez, N. López and J. R. GalanMascaros, ChemSusChem, 2017, 10, 4552-4560.

24 Y. Aratani, T. Suenobu, K. Ohkubo, Y. Yamada and S. Fukuzumi, Chem. Commun., 2017, 53, 3473-3476.

25 M. Aksoy, S. V. K. Nune and F. Karadas, Inorg. Chem., 2016, 55, 4301-4307.

26 Z. Kap, E. Ülker, S. V. K. Nune and F. Karadas, J. Appl. Electrochem., 2018, 48, 201-209.

27 A. Devadoss, A. M. Spehar-Délèze, D. A. Tanner, P. Bertoncello, R. Marthi, T. E. Keyes and R. J. Forster, Langmuir, 2010, 26, 2130-2135.

28 C. F. Hogan and R. J. Forster, Anal. Chim. Acta, 1999, 396, 13-21.

29 D. J. Kenney, T. P. Flynn and J. B. Gallini, J. Inorg. Nucl. Chem., 1961, 20, 75-81.

30 V. Marin, E. Holder and U. S. Schubert, J. Polym. Sci., Part A: Polym. Chem., 2004, 42, 374-385.

31 B. Durham, J. V. Caspar, J. K. Nagle and T. J. Meyer, J. Am. Chem. Soc., 1982, 104, 4803-4810.

32 V. Leigh, W. Ghattas, R. Lalrempuia, H. Müller-Bunz, M. T. Pryce and M. Albrecht, Inorg. Chem., 2013, 52, 5395-5402.

33 N. G. Khaligh, RSC Adv., 2012, 2, 3321-3327.

34 S. A. V. Jannuzzi, B. Martins, M. I. Felisberti and A. L. B. Formiga, J. Phys. Chem. B, 2012, 116, 14933-14942.

35 V. K. Kaushik, J. Electron Spectrosc. Relat. Phenom., 1991, 56, 273-277.

36 F. Hegner, I. Herraiz-Cardona, D. Cardenas-Morcoso, N. Lopez, J. R. GalanMascaros and S. Gimenez, ACS Appl. Mater. Interfaces, 2017, 9, 37671-37681.

37 D. J. Morgan, Surf. Interface Anal., 2015, 47, 1072-1079.

38 X. Zhou, F. Li, H. Li, B. Zhang, F. Yu and L. Sun, ChemSusChem, 2014, 7, 24532456. 